\title{
Comparative Outcome of Loop Suspensory Reconstruction and Hook Plate Fixation for Acute Type III-V Acromioclavicular Joint Dislocation
}

\section{Yu-Jui Chang}

Chang Gung Memorial Hospital Kaohsiung Branch

Wen-Yi Chou ( $\triangle$ murraychou@yahoo.com.tw)

Kaohsiung Chang Gung Memorial Hospital https://orcid.org/0000-0002-8400-3457

Jih-Yang Ko

Chang Gung Memorial Hospital Kaohsiung Branch

Hao-Chen Liu

Chang Gung Memorial Hospital Kaohsiung Branch

Ya-Ju Yang

Chang Gung Memorial Hospital Kaohsiung Branch

Ka-Kit Siu

Chang Gung Memorial Hospital Kaohsiung Branch

\section{Research article}

Keywords: Acromioclavicular joint dislocation, Hook plate, Loop suspensory reconstruction, Coracoclavicular ligament

Posted Date: April 7th, 2020

DOl: https://doi.org/10.21203/rs.3.rs-19062/v1

License: (9) This work is licensed under a Creative Commons Attribution 4.0 International License. Read Full License 


\section{Abstract}

Background Treatment options of acromioclavicular (AC) joint dislocation depend on the injury severity and the functional demand of the patient. Common surgical options include fixation across the AC joint, coracoclavicular (CC) fixation, and AC joint or CC reconstruction. However, the clinical superiority of these various procedures is controversial. This study aimed to compare the clinical and radiological outcomes of loop suspensory reconstruction and hook plate fixation for acute unstable AC joint dislocation.

Methods We retrospectively included patients with acute unstable AC joint dislocation who were treated with loop suspensory reconstruction (group I, 23 cases) or hook plate fixation (group II, 14 cases) in our hospital from January 2010 to December 2016 with a minimum follow-up period of 1 year after surgery. We assessed the clinical outcomes of blood loss during surgery, surgical duration, duration of medication required for pain relief after the primary operation, occurrence of complications, and the Constant-Murley score; radiological analysis included postoperative arthritic change and the $\mathrm{CC}$ distance discrepancy ratio (CCDR) on preoperative and final follow-up plain films.

Results The average follow-up duration was 17.0 \pm 6.1 months. The mean duration of pain-control medication usage was $121.7 \pm 174.1$ and $235.4 \pm 251.8$ days in groups I and II, respectively, significantly lower in the $\mathrm{CC}$ reconstruction group $(\mathrm{p}=0.031)$. Group I revealed a superior Constant-Murley score at the final outpatient follow-up as compared with group II $(71.7 \pm 15.8$ vs $61.1 \pm 6.7, p=0.009)$. Regarding radiographic analysis, the hook plate group had a superior residual CCDR (group I vs II $=30 \%$ vs $80 \pm 40 \%$, p 0.001 ). In addition, there were 9 cases of subluxation noted in group I and 2 cases in group II; however, 4 cases of acromion osteolysis were observed in group II.

Conclusions The principle finding of the present study was that hook plate fixation was superior to loop suspensory reconstruction in terms of CC distance maintenance. However, the loop suspensory reconstruction group demonstrated superior clinical functional outcomes and less painkiller usage. A further randomized controlled trial with a long-term follow-up period is required.

\section{Background}

Acromioclavicular (AC) joint dislocation is a common injury, accounting for approximately $10 \%$ of all shoulder girdle injuries in clinical practice [1]. It occurs more frequently in young, active populations [2]. Injuries of the AC joint are usually classified using the Rockwood classification system and according to the functional demand of the patient [3]. This classification system assesses AC injury not only in terms of separation of the AC joint, but also compromise of the coracoclavicular (CC) ligament. The CC ligament, which is formed by integration of the conoid ligament and trapezoid ligament, contributes to the vertical stability of the shoulder girdle and maintenance of the spinoclavicular angle [4]. Regarding treatment for type I and II AC joint injuries in which the CC ligament is intact, conservative treatment results in a good prognosis [3]. Advanced AC joint injuries beyond type III involve complete disruption of both the AC ligament and CC ligament with different directions of AC separation. Surgical interventions 
for type IV-VI injuries are the mainstay of treatment, although treatment for type III injuries remains controversial $[4,5]$.

Surgical interventions for advanced $A C$ injuries are usually divided into vertical CC reconstruction, horizontal AC reconstruction or a combination of both axes; however, there is no evidence of absolute superiority of one technique over another $[3,5]$. Common procedures for vertical $\mathrm{CC}$ reconstruction include CC ligament repair, Bosworth screw fixation, CC loop reconstruction using artificial materials, autograft or allograft, etc. [6]. For horizonal AC joint reconstruction, transacromial fixation is the major principle, which is accomplished using several modalities, such as Knowles pinning, Kirschner wires pinning with the tension band technique, and hook plate fixation $[2,4,6]$. Otherwise, arthroscopicassisted surgery presents an alternative intervention and also has good results $[7,8]$.

Loop suspensory reconstruction of CC ligaments is a popular modality for the treatment of $\mathrm{AC}$ joint dislocation $[3,6,9,10]$, and includes anatomic or non-anatomic reconstruction [7]. However, soft tissue damage and related blood loss, $\mathrm{CC}$ ligament calcification, clavicle osteolysis, and even aseptic foreign body reactions have been reported. In addition, involvement of the coracoid process may lead to axillary nerve compromise [11]. The hook plate, which features a trans-subacromial hook engagement under the bottom of the acromion, is commonly used nowadays due to the advantage of providing high stability of the AC joint and good functional recovery $[12,13]$. However, complications such as subacromial impingement and rotator cuff damage [14], acromion osteolysis and further acromion fracture $[15,16]$, and clavicle fracture following a retained implant [17] have been reported. The necessary second surgery for implant removal 3-6 months later is also a concern for patients and physicians.

Despite the complications, both loop suspensory reconstruction of the CC ligament and hook plate fixation are common procedures with good clinical outcomes. Although there exists comparative research regarding these two common surgical modalities in terms of postoperative outcome and complications $[18,19]$, the treatment options remain under debate. Thus, the purpose of the present study was to compare these two common procedures with regards to functional outcomes and radiological outcomes in patients with acute advanced AC joint dislocation.

\section{Methods}

\section{Patient enrollment and data collection}

Patients with advanced $\mathrm{AC}$ dislocation (type III- $\mathrm{VI}$ ) in an acute ( $\leq 4$ weeks) setting undergoing surgical intervention were enrolled for systemic analysis. Those who were treated with loop suspensory reconstruction with synthetic Mersilene tape suture plus temporary transacromion Kirschner wire or Knowles pin fixation were allocated to group I, while group II included patients treated with hook plate fixation only. Patients with the following conditions were excluded: previous shoulder surgery, preoperative shoulder stiffness, glenohumeral joint arthritis, and ipsilateral scapula, proximal humerus, coracoid, or acromion fracture. The preoperative demographic data, including age, gender, injury 
mechanism, comorbidities, and type of AC dislocation according to the Rockwood classification, were recorded.

\section{Assessment of surgical, clinical and radiographic parameters}

The surgical duration, intraoperative blood loss, occurrence of adverse events related to surgery and interval to implant removal were documented for surgical parameter comparison. Clinical outcomes assessment included the required duration of medication for pain relief and the Constant-Murley score [20], while radiological outcomes evaluation was based on comparison of the CC distance on the preoperative $\mathrm{X}$-ray versus the $\mathrm{CC}$ distance on the final follow-up plain anteroposterior film of the injured shoulder. The CC distance was measured from the uppermost point of the coracoid to the lowermost point of the clavicle. To minimize the individual difference, the ratio of the injured shoulder CC distance over the normal contralateral shoulder CC distance, which was defined as the CCDR, was adopted for accurate analysis (Fig. 1). Injured shoulders with a CCDR of 100 indicated anatomic reduction, and a CCDR less than 100 indicated a degree of over-reduction; a CCDR between 101 and 199 indicated the degree of partial loss reduction, which implied residual subluxation, and a CCDR greater than 200 indicated complete loss of reduction. Postoperative radiographic changes, including acromial osteolysis, calcification of the $\mathrm{CC}$ distance, acromion or clavicle fracture, and $\mathrm{AC}$ joint osteoarthritic change, were documented.

\section{Surgical procedure}

The superior approach with a transverse or longitudinal incision was used, and the surgery was performed under general anesthesia with the patient in a beach chair position. In the loop suspensory reconstruction group (group I), two Mersilene tape sutures were passed from the undersurface of the coracoid with a curved passer after splitting of the deltoid, and fixation of the Merselene tape sutures on the distal clavicle were performed via two bony tunnels on the remnants of the CC ligaments (conoid and trapezoid ligaments), with node-tying on the anteroinferior side of the distal clavicle after anatomic reduction of the $\mathrm{AC}$ joint. Two supplemental transacromial Kirschner wires were applied as AC joint augmentation to enforce the fixation; these were removed 6 weeks postoperatively at an out-patient clinic without secondary surgery (Fig. 2A). In the hook plate group (group II), the hook part of the plate was inserted on the posteroinferior site of the acromion after reduction of the AC joint, and fixation screws were then applied for definite fixation (Fig. 2B). After the implant had been inserted, the wound was closed layer by layer, including repair of the torn deltotrapezial fascia in both groups. The implanted hook plates were scheduled to be removed 3 to 6 months after the index surgery.

\section{Aftercare}

After the index surgery, painkillers were given to patients as required, and a shoulder sling was employed for 6 weeks in every case. Passive range of motion exercise was initiated after the day of the surgery, and passive-assisted motion started from the third week after surgery. Active motion of greater than 90 
degrees could be carried out 6 weeks later in group I after the Kirschner wires had been removed. Any motion limitation was addressed 3 months after surgery.

\section{Statistical analysis}

In this study, statistical analysis was performed using SPSS version 22.0 (Statistical Package for the Social Sciences, version 22.0; SPSS Inc.). The null hypotheses were rejected when $p$-values were $<0.05$. Continuous variables are presented as the mean and standard deviation (SD) or the mean and range. Descriptive statistics, the chi-square test, the Wilcoxon test, and the Mann-Whitney U-test were used to compare the outcomes between the two groups.

\section{Results}

\section{Patient demographics and clinical characteristics}

From January 2010 to December 2016, a total of 80 patients who sustained acute advanced acromioclavicular dislocation and underwent surgical intervention with a clinical follow-up duration of more than one year were enrolled in the retrospective analysis. Following exclusion of 33 patients who were operated upon using different surgical procedures, such as the modified Bosworth procedure, 27 patients were allocated into group I and 20 patients into group II. Four patients in group I were excluded due to ipsilateral clavicle, acromion, or proximal humerus fractures, and 6 patients were excluded due to concomitant $\mathrm{CC}$ reconstruction in the hook plate fixation group. A total of 37 patients were therefore enrolled, including 23 shoulders in the loop suspensory reconstruction group (group I) and 14 shoulders in the hook plate fixation group (group II), for final analysis (Fig. 3). The average age of the patients was 42.1 years at the time of surgery. The duration from injury to surgery was $5.8 \pm 5.9$ days in group I and 2.1 \pm 1.5 days in group II, the difference being insignificant $(p=0.082)$. The average follow-up period was $17.7 \pm 5.3$ months in group I and $16.2 \pm 7.5$ in group II, which also indicated no significant difference between groups $(p=0.156)$. The majority of injuries were sustained in traffic accidents. The distribution of injury type was 17 patients of type III, 2 patients of type IV, and 18 cases of type V (Table 1).

\section{Surgical and clinical parameter comparisons}

Group II demonstrated a significantly shorter surgical duration and less blood loss as compared with group I (101.0 \pm 38.0 vs $66.6 \pm 26.1$ minutes, $p=0.005 ; 17.5 \pm 25.6$ vs $43.8 \pm 45.6 \mathrm{ml}, p=0.015)$ (Table 2). Most of the patients did not take painkillers regularly, and the duration of painkiller usage referred to the period during which painkillers were needed intermittently. The mean duration of painkiller usage was $121.7 \pm 174.1$ and $235.4 \pm 251.8$ days in groups I and II, respectively, significantly lower in the loop suspensory reconstruction group $(p=0.031)$. Group I revealed a significantly superior Constant-Murley score at the final outpatient follow-up as compared with group II $(71.7 \pm 15.8$ versus $61.1 \pm 6.7, p=$ 0.009). Among the patients who underwent loop suspensory reconstruction, two shoulders sustained acute infections, one related to surgical site infection and the other related to pin tract infection, which was further complicated with clavicle osteomyelitis. No other complications were observed in group I. 
With regards to the hook plate group, one patient had a surgical site infection, and revision surgery with loop suspensory reconstruction was performed successfully after adequate debridement. All patients in group II underwent a second surgery for implant removal, with a mean interval of $182.2 \pm 63.3$ days, which was significantly longer than that in group I ( $109.6 \pm 145.0$ days, $p<0.001)$. The results demonstrated that hook plate fixation required a shorter surgical duration and involved lesser blood loss than loop suspensory reconstruction, but necessitated a secondary surgery and resulted in inferior clinical outcomes (Table 2).

\section{Radiological parameter comparisons}

Regarding radiographic analysis, there was no difference in the CCDR between groups before surgery $(p=$ 0.448). Both groups showed significant improvement in the CCDR after the index surgery $(p<0.001$ in both groups); however, group II presented a significantly superior reduction maintenance at the final follow-up (CCDR: group I vs group II = $130 \pm 30$ vs $80 \pm 40, p<0.001$ ). Meanwhile, the numbers of patients with residual subluxation and loss of reduction were higher in group I, but the differences were not statistically significant (Table 2). In a comparison of clinical outcome in patients with residual subluxation and anatomic/over-reduction of group I, patients with residual subluxation had significantly improved in comparison to their preoperative status $(p=0.011)$, and no significant difference was observed as compared with the subgroup of anatomic/over-reduction $(p=0.124)$ (Table 3). Furthermore, 4 patients in group II, but none in group I, revealed acromial osteolysis $(p=0.015)$. Patients who sustained acromial osteolysis had an inferior clinical outcome as compared with the acromial intact group (Table 4). Osteoarthritic change of the AC joint was observed in 3 patients in group I and 2 patients in group II, which was statistically insignificant $(p=1.000)$ (Table 2$)$. No acromial or distal clavicle fractures occurred after surgery in either group. The results implied that hook plate fixation offered a superior outcome in terms of reduction maintenance with more frequent acromial complications.

\section{Discussion}

The principle findings of the present study demonstrated that loop suspensory reconstruction resulted in superior clinical outcomes than hook plate fixation, even in patients with residual subluxation. It also yielded fewer acromial complications and no necessity for a second surgery for implant removal, although the surgical duration was greater, the intraoperative blood loss higher, and the proportion of patients with residual displacement greater (Table 2). In light of this comprehensive comparison, we recommend loop suspensory reconstruction for advanced AC dislocation owing to the superior clinical outcomes and fewer postoperative complications, despite the longer surgical duration and greater blood loss than for hook plate fixation, which did not relate to the final outcome. Acromioclavicular joint dislocation is a common injury and frequently occurs in active young males. In our series, the average age was 42.1 years, with male dominance (accounting for $81.1 \%$ ). The injury mechanism was almost always due to direct force to the $\mathrm{AC}$ joint, either in a fall or in a traffic accident in the acute setting. The optimal treatment option for acute AC joint dislocation remains controversial. Various procedures have been described for the treatment of Rockwood type III to type VI AC joint dislocation [21]. In vertical 
stability restoration, loop suspensory reconstruction is one of the mainstays of surgical treatment, with good reported results $[9,10,22]$. Regarding horizontal stability restoration, the hook plate method is appealing, because it provides a rigid fixation and offers the promise of high-stability AC joint fixation, whilst also maintaining normal biomechanical rotation between the clavicle and the scapula [23]. The advantages of this modality are the rapid stabilization and reliable reduction maintenance [24]; however, acromial complications and postoperative pain were major concerns following the surgery.

In this study, group II demonstrated a significantly shorter surgical duration and less blood loss as compared with group I ( $101.0 \pm 38.0$ vs $66.6 \pm 26.1$ minutes, $p=0.005 ; 17.5 \pm 25.6$ vs $43.8 \pm 45.6 \mathrm{ml}, p$ $=0.015)$. The result was compatible with a systemic review and meta-analysis by Alisara et al. [25], which indicated that hook plate fixation required a significantly shorter surgical duration than loop suspensory fixation, at an average of 16.21 minutes. According to a review of the literature, few studies have assessed the intraoperative blood loss in these two groups. We postulated that both the higher blood loss and the longer surgical duration in the loop suspensory reconstruction group resulted from the more extensive anterior deltoid splitting and advanced soft tissue dissection required to allow the curved passer to pass underneath the coracoid process, which is not required in hook plate fixation. However, a blood loss of around $50 \mathrm{ml}$ would not cause major complications in the general population. Regarding neurovascular injury while passing through the coracoid process, no related complications were noted in the present study. However, for patients with multiple trauma or a high anesthesia risk, hook plate fixation might be a better choice owing to the shorter durations of anesthesia and surgery. Kirschner wires or Knowles pins are common implants used to fix the AC joint as an augmentation for temporary stability, and good outcomes have been reported [26]. However, complications such as pin migration or breakage and pin-tract infection are concerns [27]. In our series, no obvious migration of Kirschner wires was noted until removal, but one patient developed pin-tract infection complicated with clavicle osteomyelitis and eventually a frozen shoulder. On the other hand, there was also one case of surgical site infection in the hook plate fixation group developing 2 weeks after the primary surgery. In terms of complications due to infection, there were no significant differences between groups.

Hook plate fixation provided a significantly greater stability of the AC joint than loop suspensory reconstruction according to CCDR analysis (Table 2). There were 9 cases of residual subluxation in group I (39.1\%) and 2 in group II (14.3\%), which was not statistically significant $(p=0.150)$. Nevertheless, there were no clinical signs or symptoms of instability, and thus no patient required further revision in either group. This indicated that soft-tissue healing of the CC space in the loop suspensory reconstruction patients could be achieved in a non-anatomic position under the permanent stability provided by nonresorptive suture materials. Meanwhile, some reports have mentioned mechanical failure of this implant, although hook plate fixation is regarded as a rigid fixation [28]. Regarding acromial osteolysis, the lateral portion of the hook plate could cause subacromial impingement; in other words, the stress on the base of acromion increases, which could further result in acromion osteolysis, possibly even complicated by fracture. This poor result usually occurs in patients with a hook plate retention of more than 1 year [17, 29]. This complication could be attributed to an inappropriate size of the implant [16, 29], or interpreted as the force concentration phenomenon due to morphological mismatch between the acromion and hook 
plate [30]. In our series, there were 4 cases of acromion osteolysis in the hook plate fixation group, although removal of the hook implant was carried out between 3 and 6 months after surgery as previously recommended $[16,17]$. The average interval between surgery and implant removal in the present hook plate fixation group was 182.2 \pm 63.3 days (range: 114-338 days), whereas the interval in the 4 cases with acromion osteolysis was 229 days. Furthermore, these 4 cases in the hook plate fixation group presented no significant improvement in the Constant-Murley score at the final follow-up (Table 4). These results showed that the longer the implant was retained, the greater the possibility of acromial osteolysis, and soft-tissue healing around the CC space was not strongly related to the duration for which the implant was retained. This finding differed from the results of a study by Eschler et al. [18], which mentioned that acromial osteolysis in the hook plate group had no influence on the final functional result.

A meta-analysis compared these two popular techniques by analysis of the Constant-Murley score (CMS), pain visual analog score (VAS), and rates of postoperative complications. The study demonstrated that loop suspensory reconstruction resulted in a higher postoperative functional score as compared with hook plate fixation, but the surgical duration was longer. There were no differences regarding the postoperative VAS or the complication rate [27]. We adopted the interval of intermittent painkiller usage for pain assessment rather than the VAS, because it reflected the duration of pain that interfered with daily life after surgery. The mean period of painkiller usage was $121.7 \pm 174.1$ and $235.4 \pm 251.8$ days in groups I and II, respectively, significantly lower in the loop suspensory reconstruction group $(p=0.031)$. In addition, the patients in group I revealed significantly superior Constant-Murley scores at the final outpatient follow-up than those in group II (71.7 \pm 15.8 versus $61.1 \pm 6.7, p=0.009)$, which revealed that loop suspensory reconstruction provides a superior clinical outcome in terms of both clinical function and pain reduction.

Limitations of the present study existed. First, the inherent weakness of the retrospective nature of the study and the limited case numbers weakened the strength of the statistical power. Second, the nonrandomized control study may have led to selection bias. Third, long-term follow-up (more than 2 years) comparison of these two groups could not be performed in the present study. However, by the combination of a literature review and comprehensive comparison in the present study, we were able to conclude a superior outcome of CC loop suspensory reconstruction over hook plate fixation.

\section{Conclusions}

Coracoclavicular loop suspensory reconstruction yielded a superior clinical outcome over hook plate fixation, even in patients with radiographic residual displacement. It also resulted in fewer acromial complications, and a second surgery was not required for implant removal, although the duration of surgery was longer, and intraoperative blood loss was greater. Following this comprehensive comparison, we recommend loop suspensory reconstruction over hook plate fixation for advanced AC dislocation due to the superior clinical outcome and fewer postoperative complications despite the longer surgical duration and greater blood loss, which were not related to the final outcome. 


\section{Abbreviations}

AC

acromioclavicular

CC

coracoclavicular

CCDR

CC distance discrepancy ratio

SD

standard deviation

CMS

Constant-Murley score

VAS

visual analog score

\section{Declarations}

\section{Ethics approval and consent to participate}

The study was approved by the ethical committees of the Chang Gung Memorial Hospital (approval number: IRB Study No. 201800532B0). The need for individual consent was waived by both committees because of the retrospective nature of the study.

\section{Consent for publication}

Not applicable

\section{Availability of data and materials}

The datasets used and/or analysed during the current study available from the corresponding author on reasonable request.

\section{Competing interests}

The authors declare that they have no competing interests.

\section{Funding}

Each author certifies that he or she has no any funding source that might pose a conflict of interest in connection with the submitted article.

\section{Authors' contributions}


YJC participated in study design, data collection and drafted the manuscript. YJY collected and analyzed the data. JYK collected and analyzed the data, and discussed the results. WYC participated in study design, critically read and revised the manuscript. HCL participated in data collection, and literature review. KKS participated in discussing the results and reviewing the literature. All authors cared patients, read and approved the final manuscript.

\section{Acknowledgements}

We would like to thank all nurses, radiologists and medical staffs for their great contributions during intraoperative cooperation, postoperative care and following up.

\section{References}

1. Babhulkar, A. and A. Pawaskar, Acromioclavicular joint dislocations. Curr Rev Musculoskelet Med, 2014. 7(1).

2. Bishop, J.Y. and C. Kaeding, Treatment of the acute traumatic acromioclavicular separation. Sports Med Arthrosc Rev, 2006. 14(4).

3. Tauber, M., Management of acute acromioclavicular joint dislocations: current concepts. Arch Orthop Trauma Surg, 2013. 133(7).

4. Willimon, S.C., T.R. Gaskill, and P.J. Millett, Acromioclavicular joint injuries: anatomy, diagnosis, and treatment. Phys Sportsmed, 2011. 39(1).

5. Epstein, D., M. Day, and A. Rokito, Current concepts in the surgical management of acromioclavicular joint injuries. Bull NYU Hosp Jt Dis, 2012. 70(1).

6. van Bergen, C.J.A., et al., New insights in the treatment of acromioclavicular separation. World $\mathrm{J}$ Orthop, 2017. 8(12).

7. Tomlinson, D.P., et al., A modified technique of arthroscopically assisted AC joint reconstruction and preliminary results. Clin Orthop Relat Res, 2008. 466(3).

8. Scheibel, M., et al., Arthroscopically assisted stabilization of acute high-grade acromioclavicular joint separations. Am J Sports Med, 2011. 39(7).

9. Morrison, D.S. and M.J. Lemos, Acromioclavicular separation. Reconstruction using synthetic loop augmentation. Am J Sports Med, 1995. 23(1).

10. Li, Q., P.L. Hsueh, and Y.F. Chen, Coracoclavicular ligament reconstruction: a systematic review and a biomechanical study of a triple endobutton technique. Medicine (Baltimore), 2014. 93(28).

11. Lo, I.K., S.S. Burkhart, and P.M. Parten, Surgery about the coracoid: neurovascular structures at risk. Arthroscopy, 2004. 20(6).

12. Koukakis, A., et al., Results using the $A O$ hook plate for dislocations of the acromioclavicular joint. Expert Rev Med Devices, 2008. 5(5).

13. Kienast, B., et al., Mid-term results after operative treatment of rockwood grade III-V acromioclavicular joint dislocations with an AC-hook-plate. Eur J Med Res, 2011. 16(2). 
14. Lin, H.Y., et al., Clavicular hook plate may induce subacromial shoulder impingement and rotator cuff lesion-dynamic sonographic evaluation. J Orthop Surg Res, 2014. 9.

15. Chiang, C.L., et al., Acromion osteolysis and fracture after hook plate fixation for acromioclavicular joint dislocation: a case report. J Shoulder Elbow Surg, 2010. 19(4).

16. Hoffler, C.E. and S.G. Karas, Transacromial erosion of a locked subacromial hook plate: case report and review of literature. J Shoulder Elbow Surg, 2010. 19(3).

17. Nadarajah, R., et al., Clavicular hook-plate: complications of retaining the implant. Injury, 2005. 36(5).

18. Eschler, A., et al., Hook plate fixation for acromioclavicular joint separations restores coracoclavicular distance more accurately than PDS augmentation, however presents with a high rate of acromial osteolysis. Arch Orthop Trauma Surg, 2012. 132(1).

19. Yoon, J.P., et al., Comparison of results between hook plate fixation and ligament reconstruction for acute unstable acromioclavicular joint dislocation. Clin Orthop Surg, 2015. 7(1).

20. Constant, C.R. and A.H. Murley, A clinical method of functional assessment of the shoulder. Clin Orthop Relat Res, 1987(214).

21. Fraser-Moodie, J.A., N.L. Shortt, and C.M. Robinson, Injuries to the acromioclavicular joint. J Bone Joint Surg Br, 2008. 90(6).

22. Carofino, B.C. and A.D. Mazzocca, The anatomic coracoclavicular ligament reconstruction: surgical technique and indications. J Shoulder Elbow Surg, 2010. 19(2 Suppl).

23. Sim, E., et al., Repair of complete acromioclavicular separations using the acromioclavicular-hook plate. Clin Orthop Relat Res, 1995(314).

24. Kienast, B., et al., Mid-term results after operative treatment of rockwood grade III-V Acromioclavicular joint dislocations with an AC-hook-plate. Eur J Med Res, 2011. 16(2).

25. Arirachakaran, A., et al., Comparison of surgical outcomes between fixation with hook plate and loop suspensory fixation for acute unstable acromioclavicular joint dislocation: a systematic review and meta-analysis. Eur J Orthop Surg Traumatol, 2016. 26(6).

26. Leidel, B.A., et al., Consistency of long-term outcome of acute Rockwood grade III acromioclavicular joint separations after K-wire transfixation. J Trauma, 2009. 66(6).

27. Horst, K., et al., Operative treatment of acute acromioclavicular joint injuries graded Rockwood III and IV: risks and benefits in tight rope technique vs. k-wire fixation. Patient Saf Surg, 2013. 7.

28. Charity RM, Haidar SG, Ghosh S, Tillu AB. Fixation failure of the clavicular hook plate- a report of three cases. J Orthop Surg (Hong Kong) 2006;14-333-5.

29. Chaudry, S.N. and M. Waseem, Clavicular hook plate: complications of retaining the implant. Injury, 2006. 37(7).

30. Yoon, J.P., et al., Morphological analysis of acromion and hook plate for the fixation of acromioclavicular joint dislocation. Knee Surg Sports Traumatol Arthrosc, 2017. 25(3).

\section{Tables}


Table 1. Demographics Characteristics

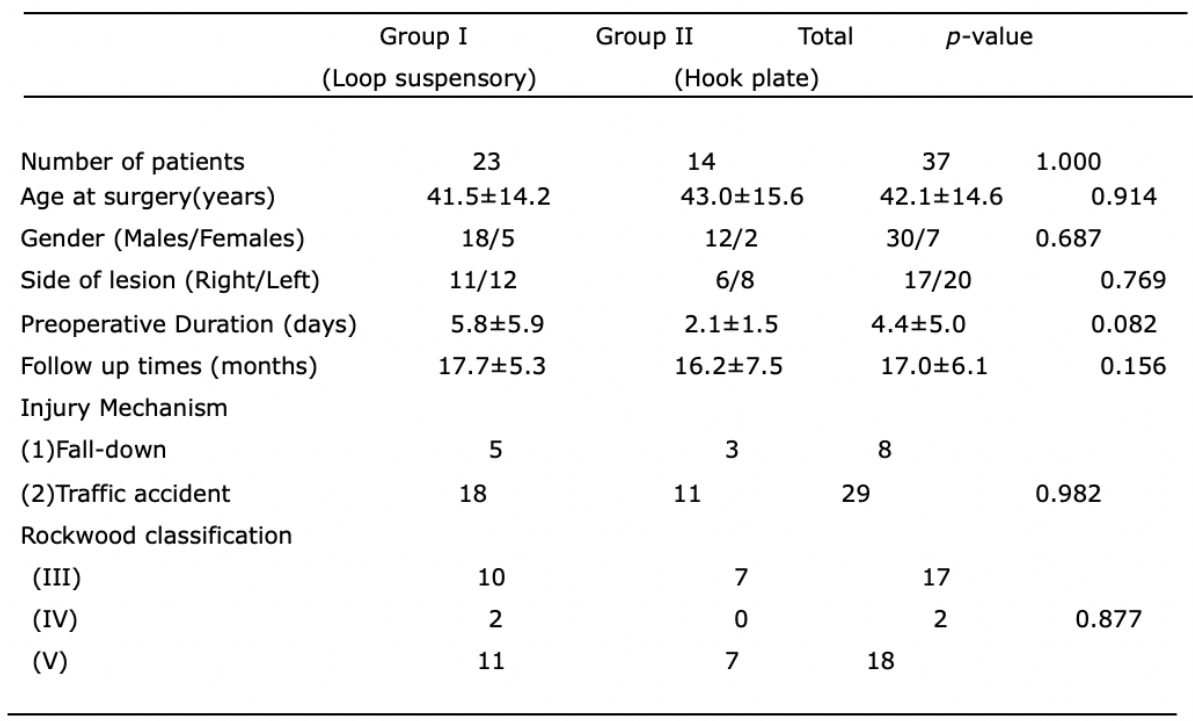

Table 2. Comparative Surgical Parameter, Clinical, and Radiological Outcomes

\begin{tabular}{|c|c|c|c|}
\hline Outcome & $\begin{array}{l}\text { Group I(Loop suspensory) } \\
\qquad(\mathrm{N}=23)\end{array}$ & $\begin{array}{l}\text { Group II(Hook plate) } \\
\qquad(\mathrm{N}=14)\end{array}$ & $p$-value \\
\hline \multicolumn{4}{|l|}{ I. Surgical parameter } \\
\hline Surgical time & $101.0 \pm 38.0$ & $66.6 \pm 26.1$ & 0.005 \\
\hline Blood loss & $43.8 \pm 45.6$ & $17.5 \pm 25.6$ & 0.015 \\
\hline \multicolumn{4}{|l|}{ Interval of implant } \\
\hline removal (days) & $109.6 \pm 145.0$ & $182.2 \pm 63.3$ & $<0.001$ \\
\hline \multicolumn{4}{|l|}{ II. Clinical parameter } \\
\hline \multicolumn{4}{|l|}{ Period of pain relief } \\
\hline medicine usage(days) & $121.7 \pm 174.1$ & $235.4 \pm 251.8$ & 0.031 \\
\hline \multicolumn{4}{|l|}{ Constant-Murley Score } \\
\hline Before treatment & $36.0 \pm 4.9$ & $35.9 \pm 3.5$ & 0.938 \\
\hline After treatment & $71.7 \pm 15.8$ & $61.1 \pm 6.7$ & 0.009 \\
\hline$p$-value & $<0.001$ & $<0.001$ & \\
\hline Infection & 2 & 1 & 1.000 \\
\hline \multicolumn{4}{|c|}{ III. Radiological parameter } \\
\hline Initial CCDR(\%) & $200.0 \pm 40.0$ & $210.0 \pm 60.0$ & 0.448 \\
\hline Final CCDR(\%) & $130.0 \pm 30.0$ & $80.0 \pm 40.0$ & $<0.001$ \\
\hline$p$-value & $<0.001$ & $<0.001$ & \\
\hline Over-Reduction & 10 & 8 & 0.420 \\
\hline Anatomic Reduction & 4 & 4 & 0.445 \\
\hline Residual Subluxation & 9 & 2 & 0.150 \\
\hline Loss of Reduction & 0 & 0 & 1.000 \\
\hline \multicolumn{4}{|l|}{ IV. Radiological finding } \\
\hline Acromion Osteolysis & 0 & 4 & 0.015 \\
\hline CC Calcification & 3 & 2 & 1.000 \\
\hline Acromion or Clavicle $\mathrm{Fr}$ & Fracture & 0 & 1.000 \\
\hline AC Joint Osteoarthritis & 3 & 2 & 1.000 \\
\hline
\end{tabular}


Table 3. Functional Outcomes Comparison between Residual Subluxation Subgroup to Anatomic/Over reduction Subgroup in Group I and to Hook Plate Group,respectively

\begin{tabular}{lccccc}
\hline Constant-Murley Score & Residual Subluxation & Anatomic/Over Reduction & $p$-value & Hook Plate & $p$-value \\
\hline Number of patients & 9 & 14 & 14 & \\
Before treatment & $35.2 \pm 4.2(28-40)$ & $36.6 \pm 5.4(26-46)$ & 0.781 & $35.9 \pm 3.5(27-41)$ & 0.926 \\
After treatment & $64.9 \pm 17.5(26-79)$ & $76.0 \pm 13.4(50-91)$ & 0.124 & $61.1 \pm 6.7(48-78)$ & 0.201 \\
p-value & 0.011 & $<0.001$ & & $<0.001$ & \\
\hline
\end{tabular}

Table 4. Functional Comparison of Hook Plate Group with or without Acromion Complications

\begin{tabular}{cccc} 
& Acromion Osteolysis & Acromion intact & $p$-value \\
\hline & $(\mathrm{N}=4)$ & $(\mathrm{N}=10)$ & \\
\hline Before treatment & $34.5 \pm 5.1$ & $36.4 \pm 0.8$ & 0.839 \\
After treatment & $57.0 \pm 4.9$ & $62.8 \pm 9.6$ & 0.454 \\
$p$-value & 0.068 & 0.005 & \\
\hline
\end{tabular}

\section{Figures}

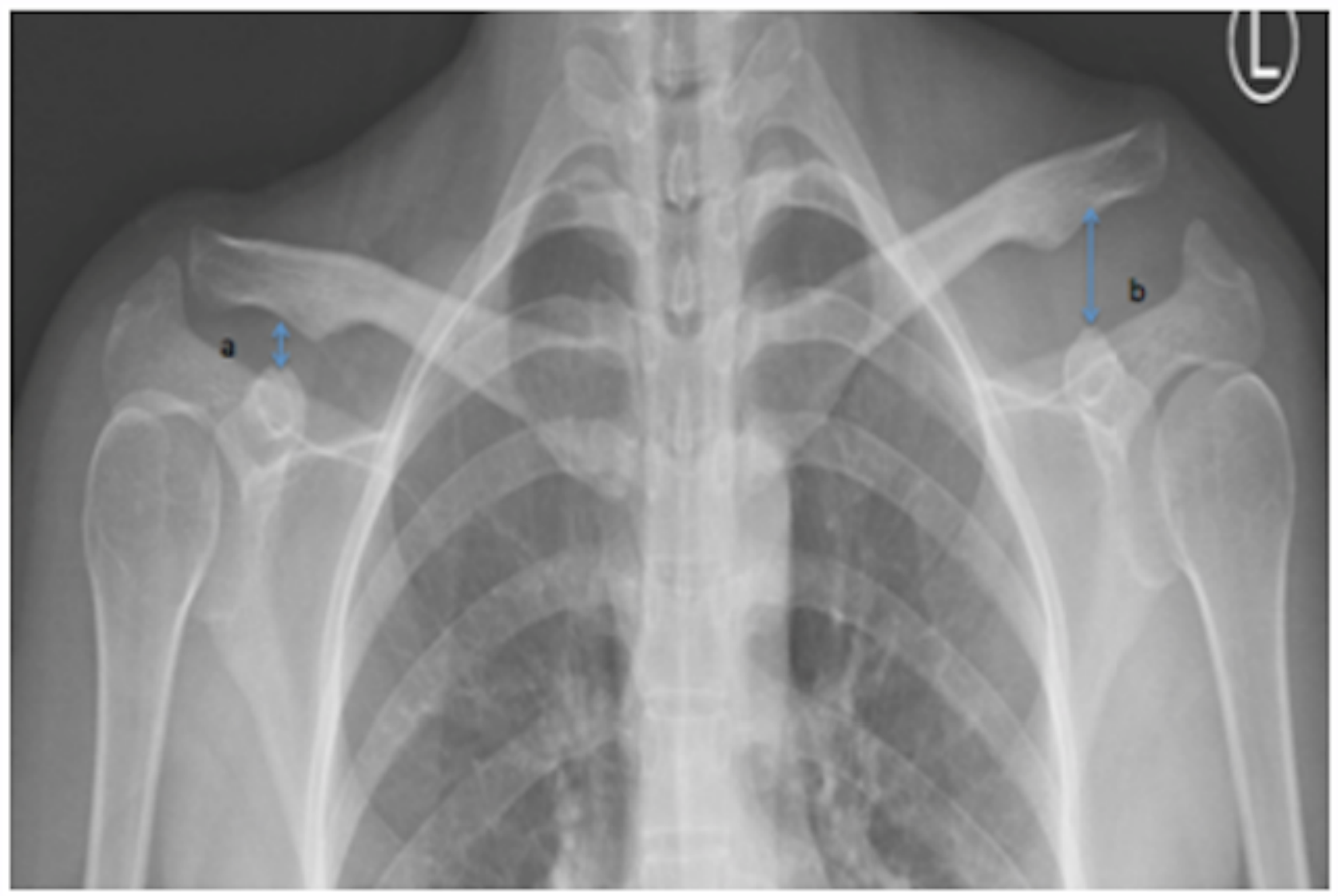

Figure 1 
The CCDR(coracoclavicular distance discrepancy ratio $=b / a$ ) is the ratio of coracoclavicular distance of injured shoulder over healthy shoulder. The $\mathrm{CC}$ distance was measured from uppermost point of coracoid to the lowest point of the clavicle in the vertical axis $(a, b)$.
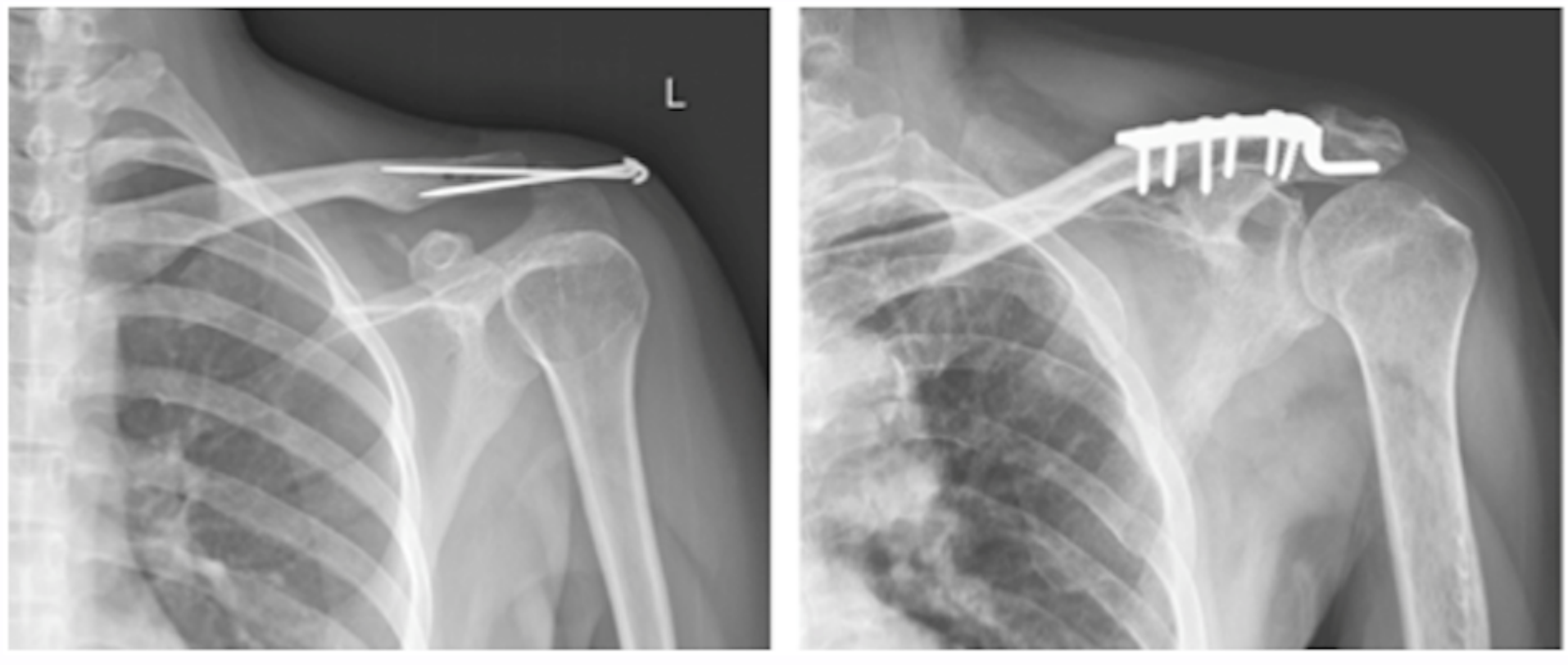

\section{Figure 2}

The Surgical Modality Used in The Two Groups. a Transarcomion k-wires fixation plus Mersilene tape reconstruction of disrupted CC ligament. b The hook plate fixation for the AC joint dislocation. 


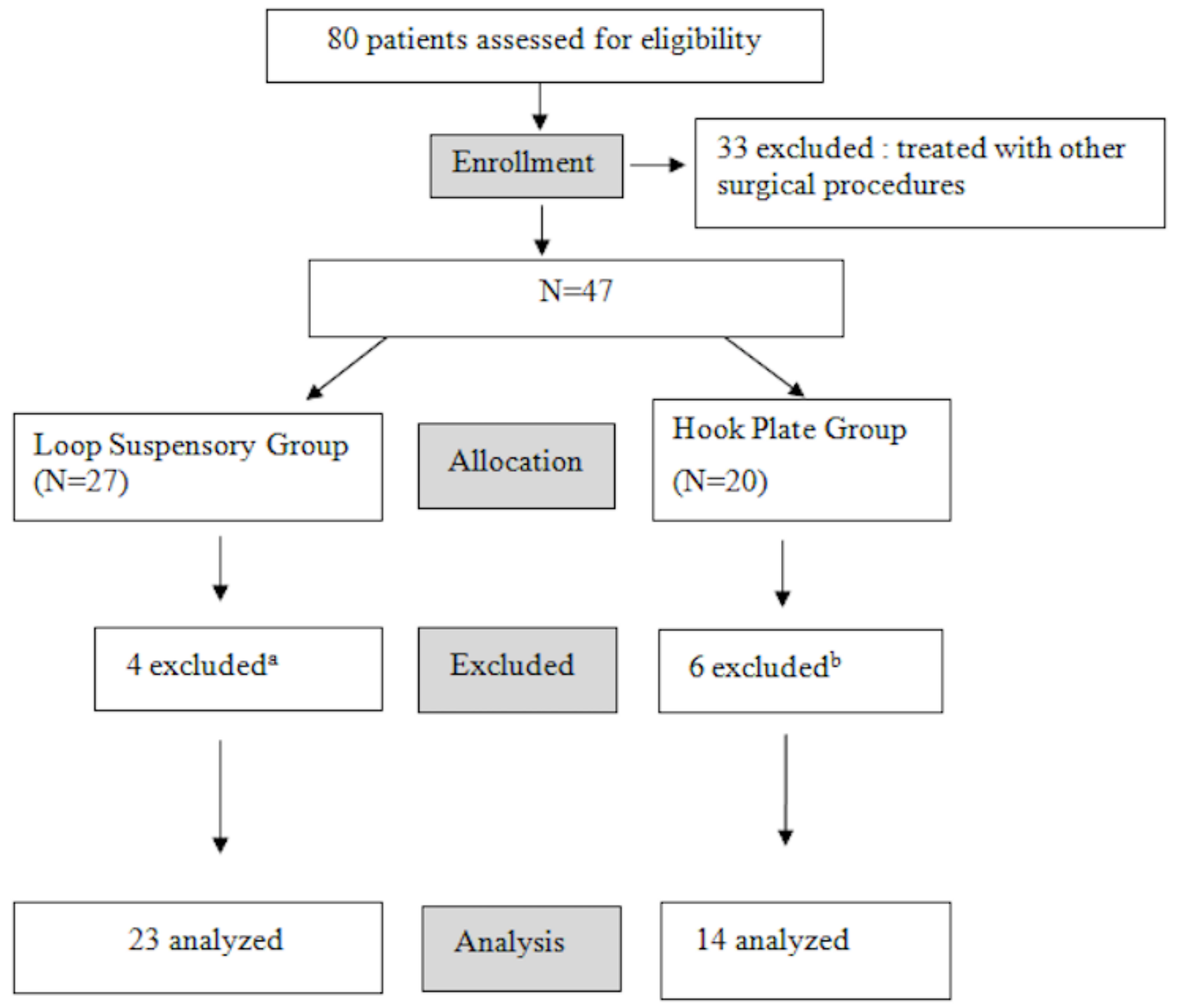

\section{Figure 3}

Flowchart of the Patients Enrollment alpsilateral clavicle, arcomion, or proximal humerus fractures bConcomitant CC reconstruction

\section{Supplementary Files}

This is a list of supplementary files associated with this preprint. Click to download.

- CompliancewithEthicalStandards.docx 